\title{
Fundamentos psicosociales de la reputación online: Propuesta de un modelo y un caso de análisis
}

\author{
Ubaldo CUESTA CAMBRA \\ Universidad Complutense de Madrid \\ ubaldo.cuesta@ccinf.ucm.es
}

Recibido: $12 / 11 / 2012$

Aceptado: 23/01/2013

\section{Resumen}

La aparición de (1) internet, (2) las redes sociales, (3) los dispositivos móviles de acceso a las redes y de (4) numerosas aplicaciones de software y de (5) plataformas específicamente diseñadas para promover la interacción social ha provocado la aparición de un fenómeno nuevo de gran relevancia: la reputación online. En los últimos años se ha comenzado a generar un corpus teórico y de investigación muy relevante en esta área (e.g. Masun y Tovey, 2012). En esta investigación, proponemos un modelo psicosocial explicativo de este fenómeno, partiendo del paradigma de la psicología evolutiva. Aplicando el método del caso único mediante monitorización online, se analiza empíricamente el sector considerado uno de los más activos dentro del entorno del social media (Online Reputation Management, 2012: web) y se plantean futuras líneas de investigación.

Palabras clave: Reputación online, psicología de la comunicación, social media, monitorización online.

\section{Psychosocial Basis of the Online Reputation: a Proposal for a Model and a Case Study}

\begin{abstract}
The appearance of (1) Internet (2) social networks (3) mobile devices access to networks and (4) numerous applications and software (5) platforms specifically designed to promote social interaction has led the emergence of a new phenomenon of great relevance: online reputation. In recent years it has begun to generate a body of theory and very relevant research in this area (eg. Masun and Tovey, 2012). In this research, we propose a psychosocial model explaining this phenomenon, based on the paradigm of evolutionary psychology. Applying the case method by monitoring online, we empirically analyzed one of the sectors considered the most active in the social media environment (Online Reputation Management: Web, 2012) and we proposed future research.

Keywords: Online reputation, communication psychology, social media, online monitoring

\section{Referencia normalizada}

CUESTA CAMBRA, Ubaldo (2013): "Fundamentos psicosociales de la reputación online: Propuesta de un modelo y un caso de análisis". Estudios sobre el mensaje periodístico. Vol. 19, Núm. especial abril, págs.: 691-700. Madrid, Servicio de Publicaciones de la Universidad Complutense.
\end{abstract}

Sumario: 1. Introducción. 2. Metodología. 3. Desarrollo. 4. Conclusiones. 5. Referencias bibliográficas.

\section{Introducción}

La psicología de la comunicación desde el paradigma de la psicología evolutiva propone que la conducta comunicativa de los sujetos consiste en relaciones mutuas de interacción triádica recíproca entre emisor, receptor y entorno social. Se trata de una conducta dinámica y pro-activa respecto a su entorno. El paradigma subraya la finalidad de la conducta comunicativa (o funcionalidad). Esta finalidad consiste, básicamente, en optimizar la obtención de refuerzos disminuyendo los castigos, siempre en un entorno competitivo, donde el componente adaptativo es esencial. Es un paradigma, pues, evolutivo, funcionalista, adaptativo. 
Desde este punto de vista evolutivo, la reputación puede ser entendida como el resultado del largo proceso de selección natural y de la "lucha" entre las especies por la supervivencia (Clippinger, 2011).

Ahora bien, las especies sociales han tenido que aprender también a desarrollar mecanismos de colaboración, no sólo de competición. Son evidentes los beneficios de colaborar o cooperar entre miembros de la misma especie para ejecutar de modo más eficaz determinadas conductas, como la lucha por la defensa del territorio o la caza.

Este equilibrio entre competitividad y colaboración entre miembros de la misma especie, es difícil de mantener. En ocasiones provoca competencias que suponen un coste excesivo el cual, a medio plazo, es perjudicial para los individuos y, lo que es más grave, para el grupo social. Este fenómeno es conocido como The Red Queen Effect (Ridley, 2003). El nombre viene extraído del personaje La reina roja de la obra de Carroll (1986) "A través del Espejo". Este personaje, como es sabido, corre y corre encolerizado sin llegar nunca a ninguna parte.

Una gran parte de los procesos anteriormente descritos están siendo reinterpretados actualmente en términos de reputación. Existen muchas definiciones de reputación, en función del modelo teórico del que parta el autor, del contexto donde se enmarque la reputación (empresarial, institucional, puramente comercial) y, especialmente, del nivel de análisis (individual, social, grupal). Sin embargo, existe un denominador común que muy pocos autores rechazarían: la reputación consiste en un conjunto de señales que facilitan la toma de decisiones a los organismos sociales para contribuir al proceso de toma de decisiones adaptativas (Dawkins, 1976).

\section{Metodología}

Este trabajo se ha articulado en torno dos áreas: en un primer momento, mediante el análisis crítico de la bibliografía más adecuada, se plantean las bases psicológicas, antropológicas y sociológicas (incluyendo los intereses económicos/empresariales) de un modelo interpretativo de la reputación online.

Dicho análisis bibliográfico incluye tanto el campo de la psicología evolutiva y la antropología como de la psicología y teoría de la comunicación. De este modo, partiendo de las principales teorías desarrolladas en estos campos (como, por ejemplo, la Teoría de la señalización de costos o CST "cost signal theory", dentro de la biología evolutiva) se construye un modelo interpretativo que introduce las aportaciones más pertinentes de estas áreas de conocimiento integrando conocimientos dispersos dentro de un corpus teórico que permita avanzar en la construcción de teorías explicativas de este nuevo e importante fenómeno.

Una vez desarrollado el modelo, se desarrolla un análisis cuantitativo basado en la metodología del caso único. Los autores ya han desarrollado otros trabajos empleando esta metodología que han descrito ampliamente en otro lugar (Cuesta, U; Gaspar, S. y Menéndez, H. (2012:287).

Mediante el empleo de motores de búsqueda propios de este tipo de monitorizaciones online (Loogic, 2012: Web) se rastrean las redes sociales del sector de automoción, concretamente motocicletas, considerado uno de los más activos en el área de la reputación social (OnLine Reputation Management, 2012: web). El periodo de 
monitorización cubrió desde el 12 de julio al 27 de agosto de 2012. Considerando que el objetivo del análisis de caso consistía en conocer el escenario global del sector, especialmente desde el punto de vista acumulativo, el periodo temporal no tiene por qué ser aleatorio. De hecho, la muestra recogida fue de tal volumen de datos que proporciona una idea muy objetiva del contenido real del discurso social del sector. Los datos recogidos fueron extraídos de foros en su totalidad, dado que el resto de las redes sociales no arrojaron datos relevantes.

Método de observación empleado: mediante plantilla de análisis de contenidos cuantificada según la metodología presentada en investigaciones similares del autor (Cuesta et. al 2010) dos personas suficientemente entrenadas en estas tareas han recogido la información durante ocho horas diarias.

Se analizaron 28 foros, 6.341 temas y 2.133 .640 visitas.

La metodología empleada para la codificación de las unidades narrativas ha sido utilizada en otras investigaciones por los autores (Cuesta y Menéndez, 2008). Consiste en una metodología cuantitativa de análisis de contenidos y de codificación mediante plantillas. La elección de dichas unidades se realizó mediante la técnica de cuerdo inter-jueces, en base a los contenidos semio-narrativos que presentaba y que remitían a las construcciones discusiones temáticas analizaban. La valoración de la valencia de las emociones mostrada en los contenidos semio-narrativos se estableció también por acuerdo inter-jueces en una escala nominal del tipo: 1=positivo, $2=$ muy positivo, $3=$ negativo, $4=$ muy negativo.

\section{Desarrollo}

La Teoría de la señalización de costos o CST (cost signal theory) dentro de la biología evolutiva, es un cuerpo de trabajo teórico que analiza la comunicación entre las personas y las instituciones. En ella se analiza si se puede esperar que los organismos con intereses en conflicto se lleguen a comunicar "honestamente". Pero también se están empezando a difundir modelos de este tipo en el ámbito de la conducta online de intercambio de archivos ilegales peer-to-peer (Lyle y Sullivan, 2007).

La reputación, en cuanto mecanismo evolutivo, consiste en un mecanismo que favorece la toma de decisiones eficaz, proporcionando la adecuada información.

En este sentido puede afirmarse que el aprendizaje cooperativo y la conducta de altruismo se basa también en un mecanismo de refuerzo, de obtención de premios. Los sujetos (y las organizaciones) se encuentran auto-motivados por el auto-interés. Por ejemplo, cuando las personas actúan como revisores en Amazon o en Yelp, en IMDB (International Movie Data Base), en Spotify o en un foro cualquiera, están actuando desinteresadamente, pero están obteniendo dos cosas: en primer lugar, una etiqueta de estatus que es socialmente muy valorada por él y en segundo lugar un placer cognitivo obtenido por la realización de la tarea en sí misma. La etiqueta de estatus es incluso reforzada por muchos sistemas digitales reputacionales, estableciendo sistemas de refuerzo social. Por ejemplo, digg.com establece jerarquías de revisores, distinguiendo en función de esas jerarquías diferentes funciones en la comunidad. Los sujetos emiten esas conductas impulsados por la anticipación de refuerzos positivos, más que por el cálculo de las consecuencias biológicas evolutivamente establecidas 
(Baytiyeh y Pfaffman, 2010). Recientemente comienza a denominarse a esta parte "oculta" las actitudes implícitas" (Kahneman, 2012) dentro de los llamados modelos duales del procesamiento de la información (Petty y Cacioppo, 1986).

La importancia de esta teoría de cara a los modelos de reputación, y muy especialmente de reputación online, consiste en dos de las afirmaciones que se derivan del modelo:

» La ruta periférica es automática, es decir, actúa de forma no-consciente. El sujeto no verbaliza los mecanismos por los que ha llegado a esa conclusión.

» La ruta periférica permite actuar de forma muy rápida.

Precisamente por los dos postulados anteriores, el libro del premio nobel Daniel Kahneman (2011) se titula Thik fast, think slow ("Pensar rápido, pensar despacio" en su traducción por la editorial al español).

La reputación actúa en gran medida de esta manera: emite determinadas señales sociales que inducen al receptor de éstas a tomar decisiones positivas y, por lo tanto, a actuar en consecuencia. Este proceso se produce por la ruta periférica, de tal manera que la reputación está actuando sobre los sujetos sin que éstos sean muy conscientes de ello, a través de mecanismos muy rápidos y procesamientos truncados.

En el ámbito de la reputación online, las decisiones se desarrollan con mucha frecuencia de forma muy rápida. El propio mundo online provoca que todo se desarrolle de manera muy dinámica, con poco tiempo para la reflexión. Algunos autores han llegado a considerar que una de las principales características que define el entorno digital es, precisamente, la de la inmediatez, la cual va muy unida a la rapidez de respuesta (Safko,2010).

La reputación online actúa, de esta manera y bajo las mencionadas circunstancias, mediante el empleo de estas rutas periféricas y empleando los mecanismos heurísticos (procesamientos truncados) a los que hacíamos referencia anteriormente.

El título del libro de Hassan Massum, uno de los mayores expertos en reputación y una de las mentes más originales, creativas y eficaces del momento, refleja muy adecuadamente esta idea: La Sociedad de la reputación: Cómo las opiniones online están reconfigurando el mundo offline (Masum, 2011).

Todo ello, refleja la manera en la cual se está produciendo la simbiosis entre el nuevo mundo digital, heredero una tecnología extraordinariamente desarrollada, y las bases biológicas y evolutivas (antropológicas, en definitiva) del ser humano. Estos mecanismos de toma de decisiones rápidas basadas en la reputación, como venimos exponiendo, se encuentran profundamente enraizados en la antropología del ser humano.

Para estos y otros autores, una de las dificultades que proporcionan las redes sociales, el mundo virtual online, consiste en el tipo de relación que se establece con los otros: en el mundo offline las habilidades de relación social, la asertividad se construye lentamente con el aprendizaje cotidiano por dos grandes mecanismos: el aprendizaje vicario, en el cual el sujeto aprende por observación de modelos determinada conducta y el modelamiento social (Cuesta, 2000; Bandura, 1986), en el cual es sujeto aprende por ensayo y error y dispensación de premios y castigos sociales (sanciones sociales). 
En cambio, en el universo virtual online, muchos de estos mecanismos no se producen. Por ejemplo, si la respuesta no verbal de un amigo a un comentario mío en $\mathrm{Fa}$ cebook me envía señales de retroinformación de desacuerdo, es muy posible que modifique el resto de mi conversación, o busque mecanismos de cooperación. Sin embargo, en el mundo online, estas claves pasan desapercibidas. O, todavía pero, estas u otras claves, son pasadas por alto, ignoradas; así, es frecuente simplemente desconectar cuando, en la red, el otro envía una retroalimentación negativa.

Esto ha ido llevando poco a poco al desarrollo de sistemas cada vez más amigables e interactivos, la llamada social interaction, donde el papel del usuario es tan relevante que se ha acuñado la frase the user is the King. Es esta interacción, este injerto entre los mundos virtuales y reales, lo que está dando lugar a esta especie de evolución por mutación a la que nos referíamos. Algunos autores (Kelly, 2010) llamaban a este proceso una hibridación de tecnología, biología, ecología, biónica y vida artificial, una especie de era neo-biológica.

Esta hibridación o simbiosis entre el mundo online y el offline, está siendo responsable de la re-configuración del mundo real por el mundo virtual y tiene uno de sus más claros exponentes en el campo de la reputación digital.

Existe una gran variedad de contextos en los cuales se produce esta interacción, mediatizados por diferentes tecnologías, distintos objetivos, empleo de software etc. Y donde el concepto de reputación, es fundamental: Apps, Videojuegos, SMS, Chats, mensajería directa tipo WhatsApp, Foros, Twitter, redes tipo Facebook o Tuenti, "recomendaciones" como Digg, Meneame, Yelp, distribuidores como Amazon...

De todos ellos, uno de los más relevantes hace referencia al área de los foros, donde la interacción entre los mundos off y on line es muy intensa dado que acumula las características propias de "la vida real" dentro de la virtual: (1) se produce un auténtico diálogo, (2) entre diferentes personas,(3) con el deseo de obtener información valiosa para (4) desarrollar adecuadamente el proceso de toma de decisiones y (5) actuar en consecuencia.

En el gráfico 1 puede verse una comparativa obtenida de la investigación cuantitativa que hemos desarrollado entre la vida real y los discursos sociales: se comparan los datos de "potenciales emisores" (en función del número de matriculaciones por marcas) y de "discursos reales" (en función de las visitas a los foros).

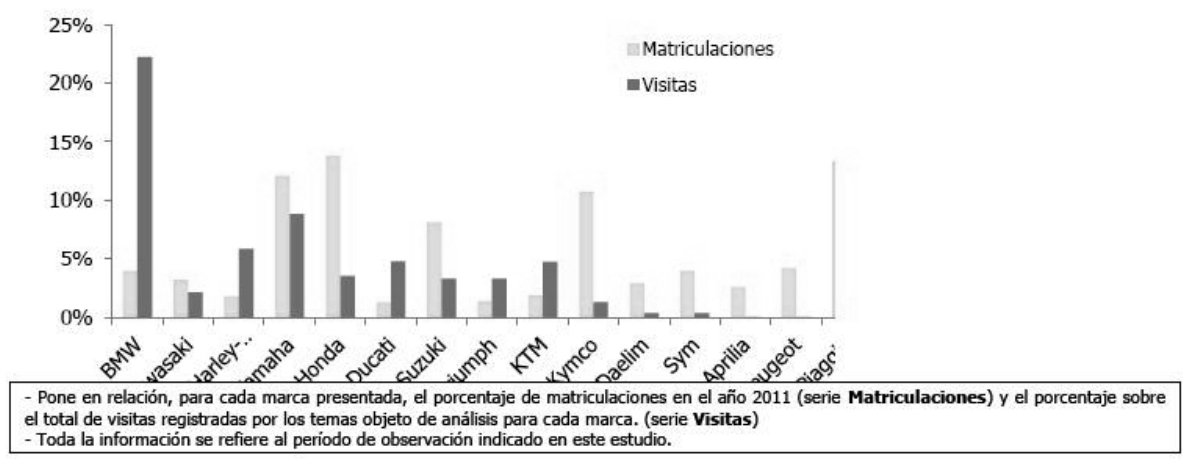

Gráfico 1: Comparativa entre potencial emisor (matrículas) y presencia en la red (visitas) 
Se añade a esto un concepto de gran valor, los foros se alzan como una de las herramientas más importantes en la gestión empresarial basada en los stkeholders y la reputación.

Muchas empresas (grandes, medianas y pequeñas) se plantean actualmente en qué medida influye en su reputación la correcta gestión de los stakeholders y hasta qué punto esta gestión afecta a la capacidad de la empresa para relacionarse adecuadamente con sus grupos de interés. En este caso, al ocuparnos de los foros, el grupo de interés más relevante, aunque no único, lo constituyen los clientes o posibles clientes, claro está.

La teoría de la gestión empresarial basada en los stakeholders afirma que éstos son la unidad de análisis de la que debe partir toda estrategia, para conseguir aportar valor añadido a todos ellos y, muy especialmente, a los más relevantes: clientes, trabajadores, accionistas, proveedores y grupos sociales próximos. Y todo ello, siempre con unos objetivos no-cortoplacistas. Este análisis debe partir del índice de reputación más relevante: la satisfacción del stakeholder. En la figura 2 puede verse la distribución de satisfacción e insatisfacción encontrada por temas de debate entre los foreros o stakeholders.

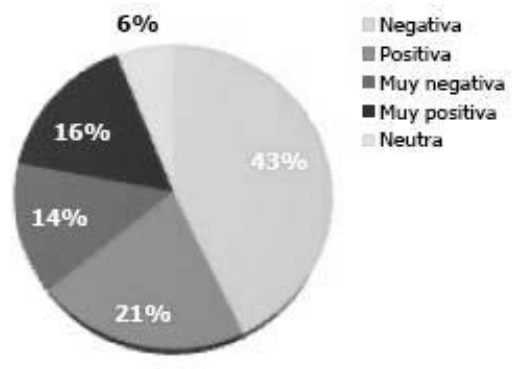

Gráfico 2: Satisfacción en porcentaje por temas entre los emisores

Tabla 1: Etiquetas del tipo de discurso social

\begin{tabular}{|c|c|c|c|}
\hline \multicolumn{4}{|c|}{ BMW } \\
\hline & Materia & Visitas & Temas \\
\hline 1 & Consulta Técnica/Manuales & 161.343 & 514 \\
\hline 2 & Experiencias/Opiniones & 86.860 & 130 \\
\hline 3 & Modelo y Versión & 48.830 & 77 \\
\hline 4 & Recambios & 37.161 & 153 \\
\hline 5 & Equipamiento Motocicleta & 19.714 & 61 \\
\hline 6 & Problema Mecánico & 18.566 & 63 \\
\hline 7 & Otros & 11.603 & 31 \\
\hline 8 & Comparativa entre Modelos & 9.465 & 17 \\
\hline 9 & Equipamiento Motorista & 5.812 & 25 \\
\hline 10 & Quedadas/Actividades & 2.674 & 8 \\
\hline
\end{tabular}


Los resultados de esta investigación demuestran cómo es posible objetivar el discurso social por más complejo que este sea. Es importante destacar que el gráfico anterior (gráfico 2) refleja el análisis de más de 6.000 conversaciones (o visitas) emitidas en la red. La tabla siguiente (tabla 1) refleja las etiquetas del tipo de discurso social: en ella se muestra el top ten de las conversaciones. Puede apreciarse no sólo la variedad de temas, sino la gran dispersión y cantidad de hilos (por emplear la terminología propia de los foros).

Especialmente ahora, con la grave crisis económica que asola el planeta, se deben poner en pié con firmeza estas ideas y concebir las empresas no como el sistema capitalista ha venido haciendo hasta ahora, como una maquinaria para ganar dinero para los financiadores (accionistas, socios capitalistas, propietarios), sino como una estructura capaz de aportar valor a todos sus interlocutores (Harris y Freeman, 2008).

Estos autores están planteando el desarrollo de un nuevo paradigma capitalista, más avanzado y mejor, que supondrá obtener beneficios sostenibles a largo plazo y más beneficiosos para el bien común global. Para autores tan relevantes como Freeman, esto supone, incluso, la aparición de una nueva narrativa sobre el mundo empresarial:

"La denominada gestión empresarial basada en la creación de valor para los stakeholders se basa en una serie de principios con los que comienza la construcción de una nueva narrativa sobre el mundo empresarial" (Freeman, 2012:315.).

Esta nueva narrativa, aparece muy mediatizada por la nueva narrativa digital, de tal manera que se produce esta interacción entre narrativas creando una potente sinergia.

\section{Conclusión}

El sistema de reputación que hemos brevemente presentado en relación con los foros de motos, pertenece a los denominados "sistemas no mediados" de reputación online. Los sistemas no mediados son aquellos en los cuales las informaciones que proporcionan los miembros de la comunidad fluyen libremente entre todos ellos, por comparación a los sistemas mediados, donde una tercera instancia los recoge, almacena, organiza y publica. Como ejemplo de sistemas no mediados se encuentran las cartas de recomendación tipo las que aparecen en LinkedIn o los informes de recomendación laboral tipo Infojob o el boca-boca en redes como Facebook o foros como los analizados de motos (y, por supuesto, de cualquier otro tema que deseemos investigar).

En cuanto a los sistemas mediados, encontramos desde sistemas muy simples del tipo espacios de revisión de consumidores (consumer review sites tipo Yelp, Amazon, eBay, TripAdvisor, RealSelf.com, Menéalo, IMDB...) hasta sistemas complejos de ratings tipo Moody.

Todos los analistas de mercado y los gerentes de grandes organizaciones están de acuerdo en que el mercado (de bienes, productos o servicios, de tipo social o comercial) se autorregula en función de las demandas agregadas de los consumidores. Hay una especie de mano invisible que controla el intercambio de bienes o servicios por otro tipo de bienes o servicios.

Cuando la información sobre productos o empresas (instituciones, organizaciones...) es costosa, la información reputacional puede incrementar la actividad de esta mano invisible ayudando a los consumidores a tomar mejores decisiones. 
De hecho, una de las definiciones clásicas de reputación consiste precisamente en esto: proporcionar señales sociales que permitan optimizar las decisiones.

En estos casos, la información reputacional actúa como una especie de mano secundaria invisible (Goldman, 2011). Ahora bien, esta mano secundaria invisible, está a su vez sometida a costos de transacción. Es costoso para los consumidores encontrar información reputacional y evaluar su credibilidad. De este modo, los sistemas de reputación frecuentemente buscan establecer su propia reputación, con lo cual se genera una especie de mano terciaria invisible.

Esta mano terciaria invisible permite al sistema reputacional ganar credibilidad entre los consumidores y actuar como una fuente creíble. Por esto motivo se generan metasistemas de intervención sobre el diálogo espontáneo de las redes en la blogosfera, con el ánimo de encauzar los discursos en el sentido deseado por la organización. También con el ánimo de conocer el discurso que se produce en la red respecto a un determinado interlocutor social (empresa, institución, o un bien o servicio particular).

Como ha demostrado esta investigación, a pesar de que el discurso social sea muy complejo e implique miles de conversaciones (visitas) que a su vez se dispersan en miles de hilos, es posible rigorizar el fenómeno y construir índices objetivos y cuantificables no sólo del tipo de discurso samionarrativo, sino de establecer índices de las valencias emocionales de ellos.

Es necesario profundizar mucho más en la construcción de modelos psicosociales rigurosos que permitan continuar desarrollando instrumentos de medida fiables y válidos, es decir, apoyados en una verdadera validez de constructo, más que en una validez concurrente como se viene haciendo hasta ahora.

\section{Referencias bibliográficas}

ARRIS, Jared y FREEMAN, R. Edward (2008): "The impossibility of the separation thesis". Business Ethics Quarterly, 18 (4), pp. 541-548.

BANDURA, A. (1986): Social Foundations of Thought and Action: A Social Cognitive Theory. Englewood Cliffs, N.J.: Prentice-Hall.

BAYTIYEH, Hoda y PFAFFMAN, Jay (2010): “Open source software: A community of altruists" Computers of Human Behavior 6, pp.1345-1354.

CARROLL, Lewis (1986): Through the looking-glass. New York, Ariel Books/Knopf.

CLARKSON, Gavin y VAN ALSTYNE, Marshall W. (2009): "The social efficiency of fairness". En Boston University School of Management Research Paper $\mathrm{N}^{\mathrm{o}}$ 2009-11. http://ssrn.com/abstract=1514137. [fecha de consulta el 10 de septiembre de 2012]

CLIPPINGER, John Henry (2011): "An Inquiry into effective reputation and rating systems": Idcubed http://idcubed.org/wp-content/uploads/2011/08/Effective_Reputation_and_Rating_Systems.pdf [fecha de consulta el 6 de septiembre]

CUESTA, Ubaldo (2000): Psicología social de la comunicación. Madrid, Cátedra.

CUESTA, Ubaldo y MENÉNDEZ, Tania (2008): Derechos humanos y prensa escrita. Madrid, Luk Comunicación, Funciva 
CUESTA, Ubaldo y MENÉNDEZ, Tania (2010): Derechos humanos y ciudadanía. Análisis de las noticias en prensa escrita. Madrid, Funciva.

CUESTA, Ubaldo, GASPAR, Sandra y MENÉNDEZ, Tania (2012): Estudio de la construcción de las identidades de género a través del programa televisivo: "Mujeres y hombres y viceversa". Icono 14, 10, 3, pp. 284-307.

DAWKINS, Richard (1976): The Selfish Gene. Oxford, Oxford University Press.

DUNBAR, Robin (1998): Grooming, Gossip, and the Evolution of Language. Harvard, Harvard University Press.

DUNBAR, Louise, B ARRENT, Robin \& LYCETT, John (2005): Evolutionary Psychology, a Beginner's Guide. Oxford, One World Books.

FARMER, Randall. y GLASS, Bryce (2010): "On Karma. Top line lessons on user reputation design (web log post)." Building web reputation systems. http://buildingreputation.com/writings/2010/02/on_karma.html [fecha de consulta: 4 de septiembre de 2012]

FREEMAN, R. Edward (2012): "La gestión empresarial basada en los stakeholders y la reputación. "Valores y ética para el siglo XXI". Madrid, BBVA, pp.389 - 409

FREEMAN, R. Edward; HARRISON Jeffrey S.; WICKS, Andrew C,; PARMA R Bidhan L. y COLLE Simone (2010): Stakeholder Theory: The state of the art. Cambridge, Cambridge University Press.

GOLDMAN, Eric (2011): “Regulating reputation.” En MASUM, Hassan y TOVEY, Mark (2011): The reputation society. How online opinions are reshaping the offline world. The MIT Press. Cambridge.

HARRIS, Jare y FREEMAN, Edward (2008): "The impossibility of the Separation Thesis.” Bussiness Ethics Quarterly, 18, 4, pp. 541-548

KAHNEMAN Daniel (2012): Think fast and slow. London, Penguin Books.

KELLY, Kevin (2010): What Technology Wants. London, Viking Penguin.

LOOGIC (2012): Herramientas de monitorización y gestión de la reputación online. http://loogic.com/herramientas-de-monitorizacion-y-gestion-de-la-reputacion-online/ [fecha de consulta: 2 octubre del 2012]

LYLE, Henry F. y SULLIVAN, Roger J. (2007): "Competitive status signaling in peerto-peer file-sharing net-works.” Evolutionary Psychology, 5, pp. 363-382.

MASUM, Hassan y TOVEY, Mark (2011): The reputation society. How online opinions are reshaping the offline world. Cambridge, The MIT Press..

MUY INTERNET (2010): “Cómo usan los jóvenes españoles Tuenti o Facebook? En Muy Internet: http://www.muyinternet.com/2010/10/19/\%C2\%BFcomo-usan-losjovenes-espanoles-tuenti-o-facebook. [fecha de consulta el 10 de septiembre del 2012]

NIELSEN WIRE (2010): en Nielsen Wire http://blog.nielsen.com/nielsenwire/[fecha de consulta: 12 de septiembre del 2012] 
OLSON, Kristina R. y SPELKE, Elizabeth S. (2008): "Foundations of cooperation in young children". Cognition 108, pp. 222-231.

ONLINE REPUTATION MANAGEMENT (2012): "Sectores con mayor número de visitas y social interaction", en: http://www.olreputation.com/es/presentacion/ [fecha de consulta: 5 de septiembre del 2011]

PETTY, Richard E., \& CACIOPPO, John T. (1986): Communication and persuasion: Central and peripheral routes to attitude change. New York, Springer-Verlag.

RIDLEY, Matt (2003): The red queen: Sex and the evolution of human nature. New York, Harper Perennial.

SAFKO, Lon (2010): The social media bible. New Jersey, John Wiley \&Sons.

SOCIAL VIDEO MARKETING (2010): "Facebook tiene una cuota de Mercado del $54 \%$ de la población total de Internet" en Social Video Marketing http://socialvideomarketing.es/facebook-tiene-una-cuota-de-mercado-del-54-de-la-poblaciontotal-de-internet/2010/11/24/ [fecha de consulta: 12 de septiembre del 2012.]

UTZ, S. (2009): "Egoboo vs. altruism: The role of reputation in online consumer communities." New Media and Society, 3, pp. 357-374.

\section{Ubaldo CUESTA CAMBRA}

Universidad Complutense de Madrid

Facultad de Ciencias de la Información

Departamento de Comunicación Audiovisual y Publicidad II

Catedrático de Universidad

ubaldo.cuesta@ccinf.ucm.es 